\title{
Immunotherapy for nonsmall cell lung cancer: a new therapeutic algorithm
}

\author{
Thierry Berghmans ${ }^{1}$, Anne-Marie Dingemans ${ }^{2,3}$, Lizza E.L. Hendriks ${ }^{2}$ and \\ Jacques Cadranel ${ }^{4}$
}

Affiliations: ${ }^{1}$ Thoracic Oncology Clinic, Institut Jules Bordet, Université Libre de Bruxelles, Brussels, Belgium. ${ }^{2}$ Dept of Pulmonary Diseases GROW - School for Oncology and Developmental Biology, Maastricht University Medical Center+, Maastricht, The Netherlands. ${ }^{3}$ Dept of Pulmonary Diseases, Erasmus Medical Center, Rotterdam, The Netherlands. "Service de Pneumologie, APHP, Hôpital Tenon and Sorbonne Université, Paris, France.

Correspondence: Thierry Berghmans, Institut Jules Bordet, Rue Héger-Bordet 1, B-1000 Brussels, Belgium. E-mail: thierry.berghmansabordet.be

@ERSpublications

Immunotherapy is a new approach for the treatment of lung cancer with impressive long-term results http://bit.ly/35GVzAn

Cite this article as: Berghmans T, Dingemans A-M, Hendriks LEL, et al. Immunotherapy for nonsmall cell lung cancer: a new therapeutic algorithm. Eur Respir J 2020; 55: 1901907 [https://doi.org/10.1183/ 13993003.01907-2019].

After a nihilistic period lasting for decades, major progress in nonsmall cell lung cancer (NSCLC) therapy were linked to the introduction of platinum-based regimens, third generation chemotherapeutic drugs and, more recently, with the discovery of oncogenic driver mutations and the development of very effective targeted therapies [1]. However, for a long time, despite known significant immune dysregulations in the lung cancer environment, clinical trials with immune therapy failed to be superior to standard therapy. Adjuvant vaccination was one of the most recent prominent failures [2]. Fundamental research led to a better knowledge of immune cell cycle control and the recognition of the role of immune checkpoints, e.g. cytotoxic T-lymphocyte associated 4 (CTLA4) and programmed death-1 (PD1) and programmed death-ligand 1 (PDL1) complexes [3,4]. This rapidly resulted in clinical application with different immune checkpoint inhibitors (ICI, monoclonal antibodies). In a 3-year period, the European Medicines Agency and the US Food and Drug Administration approved four distinct ICI for treatment of NSCLC, either in the metastatic setting or adjuvant in stage III NSCLC.

A part of the European Respiratory Society's thoracic oncology assembly research seminar entitled "New biomarkers, molecules and therapeutic sequences for NSCLC in the era of precision medicine", held in May 2019 in Paris, was dedicated to the achievements and future developments in ICI. This seminar joined international experts in the field as well as clinicians, researchers and basic scientists. We herein summarise the main points on ICI results, implementation and perspectives in NSCLC.

Several pharmaceutical companies, starting with phase I and continuing up to phase III trials, developed complex research processes with anti-PD1 and anti-PDL1. Rapidly, signals of activity associated with a good toxicity profile came from the anti-PD1 nivolumab and pembrolizumab in heavily pre-treated stage IV NSCLC [5]. Importantly, updated data showed long-term disease control, with around $10 \%$ 5-year survival without recurrent disease [6]: impressive data never reported previously in this population. After the first promising results, phase II/III trials were conducted in second line for relapsed metastatic NSCLC (table 1). Five randomised phase II-III trials testing three ICI (nivolumab, pembrolizumab and 
TABLE 1 Selected results of practice-changing immunotherapy randomised phase III trials

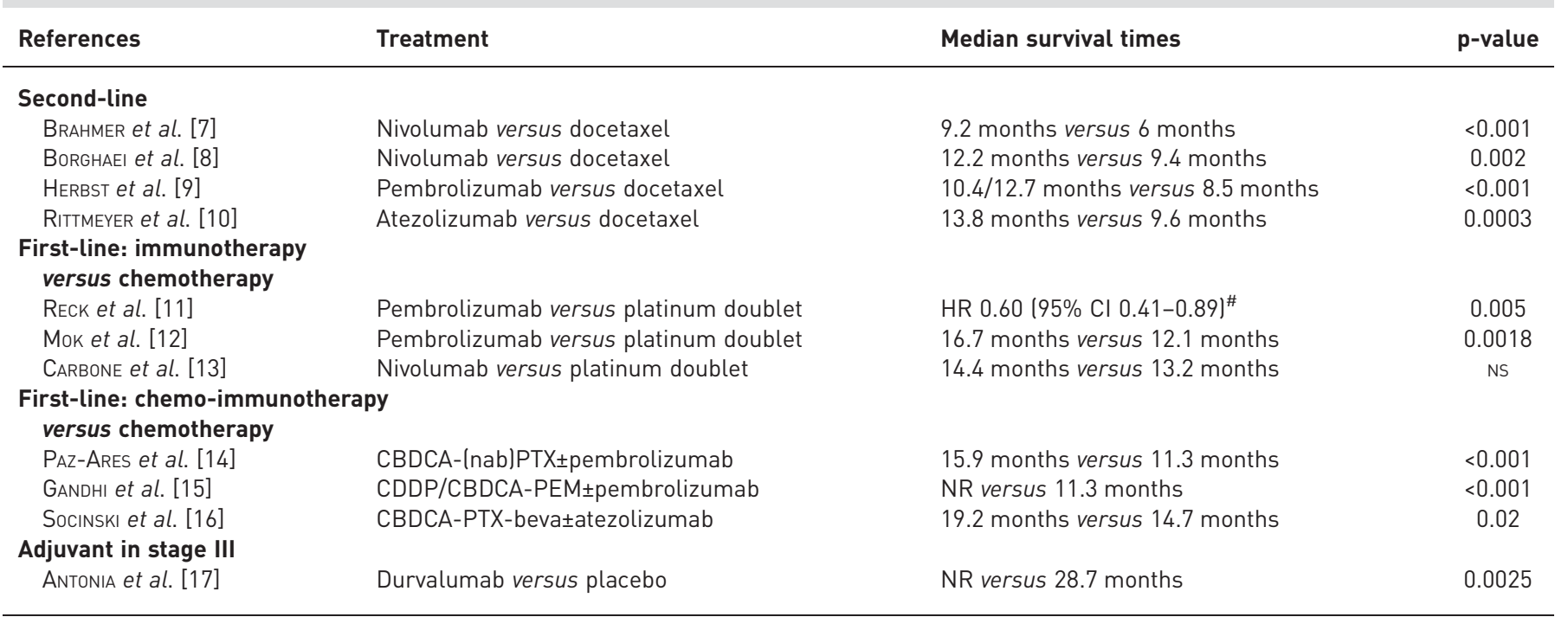

HR: hazard ratio; NS: not significant; NR: not reached; CBDCA: carboplatin; PTX: paclitaxel; CDDP: cisplatin; PEM: pemetrexed; beva: bevacizumab. ${ }^{\#}$ : no median survival available in the manuscript.

atezolizumab), all showed a clinically and statistically significant advantage over the same standard comparator docetaxel $[7-9,11,18]$. Long-term data were presented and confirmed a long-lasting effect of ICI on overall survival.

Next, those ICI were tested as first line treatment in stage IV NSCLC but showed some conflicting results (table 1). Monotherapy with pembrolizumab demonstrated significant survival superiority over platinum-based doublets only in a selected group of patients (tumour PDL1 $\geqslant 50 \%$ ) [11, 12], while this was not achieved with nivolumab [13]. Conversely, combination of pembrolizumab with chemotherapy had better activity than chemotherapy alone, without excessive supplemental toxicity $[14,15]$ regardless of the histological subtype or the PDL1 value. Furthermore, atezolizumab showed interesting activity in non-squamous NSCLC, provided bevacizumab was added to the chemotherapy combination [16], but the activity of this drug remains questionable in squamous histology.

Based on these impressive results, ICI were tested in locoregional NSCLC. The only published phase III trial demonstrated that adjuvant durvalumab in stage III NSCLC non-progressing after concomitant chemo-radiotherapy improved not only progression-free but also overall survival [17]. Also, an early clinical study showed that neo-adjuvant nivolumab before surgical resection led to $40 \%$ major pathological response [19]. In this evolving landscape, unpublished phase II and III trials showed promising results [20] with lower major pathological response rates in immunotherapy alone regimens $(22 \%$ for atezolizumab in LCM3, and 31\% for nivolumab with/without ipilimumab in NEOSTAR) than for chemo-immunotherapy regimens $(77 \%$ for chemotherapy plus nivolumab in NADIM, and 64\% for chemotherapy plus atezolizumab). Currently, three large phase III trials are conducted in completely resected stage IB-IIIA NSCLC testing pembrolizumab, nivolumab or durvalumab in an adjuvant strategy. The best sequence for adding immunotherapy to surgery, before or after resection, as well as the integration with chemotherapy needs to be clarified.

As thoracic oncologists did for targeted therapies, clinicians have to learn managing specific toxicities related to the mechanism of action of anti-PD1/PDL1 ICI. Despite some preferential sites being documented, immune-related adverse events may reach any organ at any time, which is different from chemotherapy and targeted therapies. In most of the randomised trials [7-18], ICI monotherapy is well tolerated (few grade 3-5 adverse events) and discontinuation of treatment for immune-related adverse events is around 5\%, while combinations of anti-PD1/PDL1 and anti-CTL4, such as nivolumabipilimumab, are associated with up to $10 \%$ grade 3-5 toxicity. Immune-related adverse events such as hypo/hyperthyroidism are observed in up to $10-15 \%$ of the patients and might occur as late toxicity; otherwise, the most frequent immune-related adverse events are those affecting the lung, colon, skin and liver, that in some instances could be life-threatening, as well as those considered to be more unusual, such as myocarditis or hypopituitarism. Furthermore, renal failure and interstitial nephritis are potentially problematic in patients receiving ICI-chemotherapy combinations (platinum-pemetrexed). This implies 
active detection and management by the thoracic oncologist in conjunction with organ specialists, as well as the conduct of dedicated clinical trials.

These major achievements led to substantial modifications in the therapeutic algorithm for stage III-IV NSCLC and currently, most of those patients are receiving anti-PD1/PDL1 ICI in first or eventually second line and adjuvant after concomitant chemoradiotherapy, as currently reported in the 2019 ESMO guidelines [21].

However, numerous questions remain, and were approached in the seminar during fruitful discussions (table 2). Faced with expensive drugs, it is important to define the patients who will most benefit, knowing that up to $30 \%$ may present with early progression and/or hyperprogression. Only one validated predictive biomarker is standardised and routinely available, but the accuracy of PDL1 is not sufficient for an individual prediction test in most instances. Other biomarkers, including tumour mutational burden, specific molecular alterations (SKT11, KEAP1) or some immune signatures, are undergoing testing with interesting preliminary results that should be confirmed in prospective studies [29, 30]. A second important question is regarding the management of brain metastases (BM). In almost all randomised trials, patients were only eligible if BM were previously treated and stable. Very limited data [31] suggested that ICI are active on BM, needing further confirmation. Lastly, the best therapeutic algorithm remains to be defined: is an ICI-chemotherapy regimen more active than ICI in the PDL $1 \geqslant 50 \%$ subgroup? Is maintenance chemotherapy necessary in those treated with ICI-chemotherapy, or is maintenance ICI sufficient? Which is the role of anti-CTLA4 antibodies? Is combination of ICI (anti-PD1/PDL1 plus anti-CTLA4) an interesting option, may other antigen-delivering agents (vaccination, radiotherapy, targeted drugs, etc.) improve ICI effectiveness? Can ICI be delivered to initially contra-indicated (HIV,

TABLE 2 Summary of some important questions in nonsmall cell lung cancer (NSCLC) immunotherapy

\begin{tabular}{|c|c|c|c|}
\hline Population & $\begin{array}{l}\text { Incidence in } \\
\text { NSCLC patients }\end{array}$ & Summary of available data & Conclusion \\
\hline ECOG PS $\geqslant 2[22]$ & Up to $25 \%$ & $\begin{array}{l}\text { - Limited data, often retrospective } \\
\text { - Limited benefit of monotherapy ICI } \\
\text { - ORR around } 11-12 \% \\
\text { - Median overall survival around } 3.6-5.4 \text { months, } \\
\text { in PDL1 TPS } \geqslant 50 \% 16.6 \text { months }\end{array}$ & $\begin{array}{l}\text { - Less response and reduced survival duration } \\
\text { - } \mathrm{ICI} \text { cannot be endorsed in this population } \\
\text { without caution }\end{array}$ \\
\hline Elderly [24] & $\begin{array}{l}\text { Trials }<10 \% \\
\quad \text { Daily } \\
\text { practice } 27 \%\end{array}$ & $\begin{array}{l}\text { - Also ICI benefit for overall survival in elderly } \\
\text { patients but increased risk for } \\
\text { immune-related adverse events }\end{array}$ & - $\mathrm{ICl}$ can be given to older patients \\
\hline $\begin{array}{l}\text { Auto-immune } \\
\text { disorders [25] }\end{array}$ & $14-25 \%$ & $\begin{array}{l}\text { - Mainly retrospective data } \\
\text { - Higher rate of immune-related adverse events } \\
\text { in those with auto-immune disorder compared } \\
\text { to those without } \\
\text { - Time to immune-related adverse events shorter } \\
\text { in those with auto-immune disorder } \\
\text { - Survival not different }\end{array}$ & $\begin{array}{l}\text { - ICI can be given to patients with } \\
\text { auto-immune disorders, especially those } \\
\text { without immunosuppressive medication } \\
\text { but at increased risk of immune-related } \\
\text { adverse events }\end{array}$ \\
\hline Brain metastases [26] & $\begin{array}{l}\text { Trials } 9-17.5 \% \\
\text { Daily } \\
\text { practice } 25 \%\end{array}$ & $\begin{array}{l}\text { - Trials: conflicting data } \\
\text { - Real world: conflicting data. If corrected for ECOG PS } \\
\text { and baseline steroids no deleterious effect of brain } \\
\text { metastases }\end{array}$ & $\begin{array}{l}\text { - } \mathrm{ICl} \text { can be given to patients with asymptomatic } \\
\text { brain metastases in non-eloquent areas } \\
\text { with close imaging follow-up }\end{array}$ \\
\hline Driver mutations [25] & Up to $20-25 \%$ & $\begin{array}{l}\text { - Trials: no benefit for EGFR/ALK+ patients when } \\
\text { treated with monotherapy ICl, suggestions benefit } \\
\text { for carboplatin/paclitaxel/beva/atezolizumab } \\
\text { - Retrospective data: in drivers associated } \\
\text { with non-smoking, no benefit monotherapy ICI }\end{array}$ & $\begin{array}{l}\text { - } \mathrm{ICI} \text { are only option in driver-mutated patients } \\
\text { when other treatment lines are exhausted }\end{array}$ \\
\hline HIV $[27,28]$ & Not specified & $\begin{array}{l}\text { - Review and phase I data: ICI seem safe in HIV, } \\
\text { and also beneficial } \\
\text { - No pejorative effect on viral load and CD4 cell count }\end{array}$ & - ICl may be an option in HIV patients \\
\hline
\end{tabular}


chronic viral infection, auto-immune disease, renal insufficiency) and other specific populations (poor performance status, elderly, etc.)? What is the optimal duration of immunotherapy and how do we select patients in whom ICI can be stopped without resulting in disease progression? What is the role of rechallenge ICI? How can hyperprogressive patients be identified, and does hyperprogression exist in chemotherapy-ICI treated patients? What are, besides PDL1, biomarkers that can select patients for ICI treatment (or that can identify patients that should not receive ICI at all)? Can patients be re-treated at disease progression when ICI was previously stopped and patients benefited? Is the occurrence of immune-related adverse events a possible prognostic and/or predictive marker of ICI efficacy?

Despite reproducible positive results, only a minority $(<20 \%)$ of the patients derived a very long-term benefit from ICI immunotherapy and most will progress at any time during treatment. Different strategies are under development and tested in clinical trials, such as new targets, bispecific antibodies, add-in of antigen-delivering drug/technique, combinations with radiotherapy, etc. [32].

In conclusion, immunotherapy with anti-PD1/PDL1 antibodies profoundly modified our vision in treating locally advanced and metastatic NSCLC and it will probably modify the treatment of earlier disease NSCLC. Currently, immunotherapy alone (pembrolizumab) or in combination with chemotherapy (pembrolizumab or atezolizumab) is standard of care for first-line therapy in stage IV NSCLC, while new immunotherapy combinations such as nivolumab-ipilimumab may be another option in a near future. Durvalumab is now the first immunotherapy validated for adjuvant treatment in stage III NSCLC non-progressing after concomitant radiochemotherapy. Many questions remain unresolved and should be clarified in the near future. Those are of importance when considering the financial impact of these drugs if we aim for sustainable, viable health systems.

Conflict of interest: T. Berghmans has nothing to disclose. A-M. Dingemans reports institutional fees for advisory board work and/or lectures from Roche, Eli Lilly, Boehringer Ingelheim, AstraZeneca, Pfizer, BMS, Amgen, Novartis, MSD, Takeda and Clovis, research support from BMS and AbbVie. L.E.L. Hendriks reports institutional fees for advisory board work from Boehringer Ingelheim, BMS and Lilly, travel support from Roche and BMS, grants from Roche and Boehringer Ingelheim, grants and institutional funding for a mentorship programme from AstraZeneca, personal fees for educational webinars from Quadia, outside the submitted work. J. Cadranel reports grants and personal fees from AstraZeneca, Novartis and Pfizer, and personal fees from Roche, MSD and BMS, outside the submitted work.

\section{References}

1 Hirsch FR, Scagliotti GV, Mulshine JL, et al. Lung cancer: current therapies and new targeted treatments. Lancet 2017; 389: 299-311.

2 Vansteenkiste JF, Cho BC, Vanakesa T. Efficacy of the MAGE-A3 cancer immunotherapeutic as adjuvant therapy in patients with resected MAGE-A3-positive non-small-cell lung cancer (MAGRIT): a randomised, double-blind, placebo-controlled, phase 3 trial. Lancet Oncol 2016; 17: 822-835.

3 Leach DR, Krummel MF, Allison JP. Enhancement of antitumor immunity by CTLA-4 blockade. Science 1996; 271: 1734-1736.

4 Chen DS, Irving BA, Hodi FS. Molecular pathways: next-generation immunotherapy - inhibiting programmed death-ligand 1 and programmed death-1. Clin Cancer Res 2012; 18: 6580-6587.

5 Berghmans T, Grigoriu B, Sculier JP, et al. Les inhibiteurs des freins immunitaires (anticorps anti-PD1 et anti-PDL1), une nouvelle arme thérapeutique dans les cancers bronchiques non à petites cellules [Immune checkpoint inhibitors (antibodies to PD1 and PD-L1), a new therapeutic weapon against non-small cell bronchial carcinoma]. Rev Mal Respir 2018; 35: 197-205.

6 Gettinger S, Horn L, Jackman D, et al. Five-year follow-up of nivolumab in previously treated advanced non-small-cell lung cancer: results from the CA209-003 study. J Clin Oncol 2018; 36: 1875-1884.

7 Brahmer J, Reckamp KL, Baas $\mathrm{P}$, et al. Nivolumab versus docetaxel in advanced squamous-cell non-small-cell lung cancer. N Engl J Med 2015; 373: 123-135.

8 Borghaei H, Paz-Ares L, Horn L, et al. Nivolumab versus docetaxel in advanced nonsquamous non-small-cell lung cancer. N Engl J Med 2015; 373: 1627-1639.

9 Herbst RS, Baas P, Kim D-W, et al. Pembrolizumab versus docetaxel for previously treated, PD-L1-positive, advanced non-small-cell lung cancer (KEYNOTE-010): a randomised controlled trial. Lancet 2016; 387: $1540-1550$.

10 Rittmeyer A, Barlesi F, Waterkamp D, et al. Atezolizumab versus docetaxel in patients with previously treated non-small-cell lung cancer (OAK): a phase 3, open-label, multicentre randomised controlled trial. Lancet 2017; 389: 255-265.

11 Reck M, Rodríguez-Abreu D, Robinson AG, et al. Pembrolizumab versus chemotherapy for PD-L1-positive non-small-cell lung cancer. N Engl J Med 2016; 375: 1823-1833.

12 Mok TSK, Wu YL, Kudaba I, et al. Pembrolizumab versus chemotherapy for previously untreated, PD-L1-expressing, locally advanced or metastatic non-small-cell lung cancer (KEYNOTE-042): a randomised, open-label, controlled, phase 3 trial. Lancet 2019; 393: 1819-1830.

13 Carbone DP, Reck M, Paz-Ares L, et al. First-line nivolumab in stage IV or recurrent non-small-cell lung cancer. N Engl J Med 2017; 376: 2415-2426.

14 Paz-Ares L, Luft A, Vicente D, et al. Pembrolizumab plus chemotherapy for squamous non-small-cell lung cancer. N Engl J Med 2018; 379: 2040-2051. 
15 Gandhi L, Rodríguez-Abreu D, Gadgeel S, et al. Pembrolizumab plus chemotherapy in metastatic non-small-cell lung cancer. N Engl J Med 2018; 378: 2078-2092.

16 Socinski MA, Jotte RM, Cappuzzo F, et al. Atezolizumab for first-line treatment of metastatic nonsquamous NSCLC. N Engl J Med 2018; 378: 2288-2301.

17 Antonia SJ, Villegas A, Daniel D, et al. Overall survival with durvalumab after chemoradiotherapy in stage III NSCLC. N Engl J Med 2018; 379: 2342-2350.

18 Fehrenbacher L, Spira A, Ballinger M, et al. Atezolizumab versus docetaxel for patients with previously treated non-small-cell lung cancer (POPLAR): a multicentre, open-label, phase 2 randomised controlled trial. Lancet 2016; 387: 1837-1846.

19 Forde PM, Chaft JE, Smith KN, et al. Neoadjuvant PD-1 blockade in resectable lung cancer. N Engl J Med 2018; 378: 1976-1986.

20 Vansteenkiste J, Wauters E, Reymen B, et al. Current status of immune checkpoint inhibition in early-stage NSCLC. Ann Oncol 2019; 30: 1244-1253.

21 Planchard D, Popat S, Kerr K, et al. Metastatic non-small cell lung cancer: ESMO Clinical Practice Guidelines for diagnosis, treatment and follow-up. Ann Oncol 2018; 29: Suppl. 4, iv192-iv237. Date last updated 18 Sept 2019; update available from: www.esmo.org/Guidelines/Lung-and-Chest-Tumours/Metastatic-Non-Small-Cell-Lung-Cancer

22 Middleton G, Brock K, Summers Y, et al. Pembrolizumab in performance status 2 patients with non-small cell lung cancer (NSCLC): Results of the PePS2 trial. Ann Oncol 2018; 29: mdy292.007.

23 Ricciuti B, Dahlberg S, Adeni A, et al. Immune checkpoint inhibitor outcomes for patients with non-small-cell lung cancer receiving baseline corticosteroids for palliative versus nonpalliative indications. J Clin Oncol 2019; 37: 1927-1934.

24 Remon J, Vilariño N, Reguart N. Immune checkpoint inhibitors in non-small cell lung cancer (NSCLC): Approaches on special subgroups and unresolved burning questions. Cancer Treat Rev 2018; 64: 21-29.

25 Remon J, Hendriks L, Aspeslagh S, et al. Is there room for immune checkpoint inhibitors in patients who have NSCLC with autoimmune diseases? J Thor Oncol 2019; 14: 1701-1703.

26 Hendriks LEL, Henon C, Auclin E, et al. Outcome of patients with non-small cell lung cancer and brain metastases treated with checkpoint inhibitors. J Thor Oncol 2019; 14: 1244-1254.

27 Uldrick TS, Gonçalves PH, Abdul-Hay M, et al. Assessment of the safety of pembrolizumab in patients with HIV and advanced cancer-a phase 1 study. JAMA Oncol 2019; 5: 1332-1339.

28 Cook MR, Kim C. Safety and efficacy of immune checkpoint inhibitor therapy in patients with HIV infection and advanced-stage cancer: a systematic review. JAMA Oncol 2019; 5: 1049-1054

29 Gandara DR, Paul SM, Kowanetz M, et al. Blood-based tumor mutational burden as a predictor of clinical benefit in non-small-cell lung cancer patients treated with atezolizumab. Nat Med 2018; 24: 1441-1448.

30 Ayers $\mathrm{M}$, Lunceford J, Nebozhyn $\mathrm{M}$, et al. IFN- $\boldsymbol{\gamma}$-related mRNA profile predicts clinical response to PD-1 blockade. J Clin Invest 2017; 127: 2930-2940.

31 Goldberg SB, Gettinger SN, Mahajan A, et al. Pembrolizumab for patients with melanoma or non-small-cell lung cancer and untreated brain metastases: early analysis of a non-randomised, open-label, phase 2 trial. Lancet Oncol 2016; 17: 976-983.

32 Villanueva N, Bazhenova L. New strategies in immunotherapy for lung cancer: beyond PD-1/PD-L1. Ther Adv Respir Dis 2018; 12: 1753466618794133. 